\title{
Narrative review of cytoreductive surgery and intraperitoneal chemotherapy for peritoneal metastases in ovarian cancer
}

\author{
Thomas Boerner, Oliver Zivanovic, Dennis S. Chi \\ Gynecology Service, Department of Surgery, Memorial Sloan Kettering Cancer Center, New York, NY 10065, USA \\ Contributions: (I) Conception and design: All authors; (II) Administrative support: None; (III) Provision of study materials or patients: None; (IV) \\ Collection and assembly of data: All authors; (V) Data analysis and interpretation: All authors; (VI) Manuscript writing: All authors; (VII) Final \\ approval of manuscript: All authors. \\ Correspondence to: Dennis S. Chi, MD. Gynecology Service, Department of Surgery, Memorial Sloan Kettering Cancer Center, 1275 York Avenue, \\ New York, NY 10065, USA. Email: chid@mskcc.org.
}

\begin{abstract}
Due to numerous factors, such as no specific symptoms and ineffective screening to identify premalignant or early-stage disease, most patients with ovarian cancer present with advanced-stage disease and overt peritoneal metastases. Currently, the most effective treatment for these patients is complete cytoreductive surgery with systemic platinum/taxane-based chemotherapy. Over the past few decades, many researchers have evaluated the use of postoperative normothermic intraperitoneal (NIPEC) and intraoperative hyperthermic intraperitoneal chemotherapy (HIPEC) delivery as additional treatment modalities. Here, we will review the current status and future directions for these treatment strategies in the management of ovarian cancer. Most of the studies in this area of research have been retrospective in nature, limited by heterogeneous patient populations and large variance in chemotherapeutic regimens used, leading to mixed results and difficulties in evaluating the clinical impact of the data. More mature data from prospective trials are lacking, and IP therapy for advanced ovarian cancer should still be considered an investigational approach and evaluated only in clinical trials; the exception is for patients with stage III disease who undergo interval debulking surgery after neoadjuvant chemotherapy, for whom HIPEC can be considered in selected patients as part of first-line therapy.
\end{abstract}

Keywords: Intraperitoneal chemotherapy; hyperthermic intraperitoneal chemotherapy; cytoreductive surgery; ovarian cancer; peritoneal metastases

Submitted Jul 13, 2020. Accepted for publication Sep 03, 2020.

doi: 10.21037/jgo-20-274

View this article at: http://dx.doi.org/10.21037/jgo-20-274

Ovarian cancer accounts for approximately $4 \%$ of worldwide cancer incidence and mortality among women (1). It is the seventh most common cancer in women and is responsible for more than 150,000 deaths per year worldwide (2). Due to the lack of an effective screening test, most patients present with advanced disease, with overt peritoneal metastases and concurrent ascites, which account for the high mortality rate of this malignancy (3).

We present the following article on cytoreductive surgery and intraperitoneal (IP) chemotherapy for the treatment of peritoneal metastases in ovarian cancer in accordance with the Narrative Review Checklist (available at http://dx.doi. org/10.21037/jgo-20-274). Clinical trial data were obtained via PubMed and SCOPUS searches using key terms, including the following: intraperitoneal chemotherapy; hyperthermic intraperitoneal chemotherapy; cytoreductive surgery; ovarian cancer; peritoneal metastases.

\section{Postoperative IP chemotherapy for ovarian cancer}

Due to its predilection for the peritoneal cavity, researchers have performed numerous clinical trials to evaluate the benefit of IP drug delivery as a primary treatment modality 
for advanced ovarian cancer. The studies reported a pharmacologic advantage for IP versus intravenous (IV) delivery of chemotherapy, with improved chemotherapy absorption and susceptibility of cancer cells, longer persistence in the peritoneal cavity, and enhanced chemotherapeutic doses, while limiting systemic toxic side effects (4). Among patients with recurrent disease, who have failed prior chemotherapy, the addition of hyperthermic intraperitoneal chemotherapy (HIPEC) may provide further benefit in overcoming some of the underlying chemoresistance.

The findings from 3 randomized trials that evaluated postoperative IP treatment have supported the incorporation of IP chemotherapy into the ovarian cancer treatment armamentarium (5-7). In the Gynecologic Oncology Group (GOG)-172 study by Armstrong et al., patients who underwent primary debulking surgery and achieved optimal cytoreduction (residual tumor $\leq 1 \mathrm{~cm}$ ) for stage III ovarian, fallopian tube, or peritoneal cancer were randomized to receive either IV paclitaxel $\left(135 \mathrm{mg} / \mathrm{m}^{2}\right)$ over a 24-hour period on day 1 and IV cisplatin $\left(75 \mathrm{mg} / \mathrm{m}^{2}\right)$ on day 2, or IV paclitaxel over a 24-hour period on day 1 $\left(135 \mathrm{mg} / \mathrm{m}^{2}\right)$ and IP cisplatin $\left(100 \mathrm{mg} / \mathrm{m}^{2}\right)$ on day 2 followed by IP paclitaxel $\left(60 \mathrm{mg} / \mathrm{m}^{2}\right)$ on day 8 of a 3 -week cycle. Due to severe toxicity, however, only $42 \%$ of the patients randomized to postoperative IV/IP treatment completed all 6 planned postoperative cycles. The rest of the patients crossed over to IV treatment only. Regardless, progressionfree survival (PFS) and overall survival (OS) improved with IP treatment. The use of IV/IP chemotherapy was associated with a significantly increased PFS and OS compared to the IV-only treatment (23.8 vs. 18.3 months, respectively, $\mathrm{P}=0.05$; and 65.6 vs. 49.7 months, respectively, $\mathrm{P}=0.03$ ) (6). A retrospective analysis by Suidan $e t$ al. reported similar findings of improved PFS and OS with IP therapy regardless of number of cycles (8). The authors analyzed 201 patients with stage III-IV epithelial ovarian cancer who underwent primary debulking surgery and achieved optimal cytoreduction followed by 1 or more cycles of IV/IP chemotherapy and were not able to detect a survival benefit for patients receiving 5-6 cycles versus 3-4 cycles or even $1-2$ cycles. Discontinuation of IV/IP therapy was most often associated with treatment-related toxicity (77\%).

In 2019, however, Walker et al. reported the results of the GOG-252 study, a very large phase 3 randomized, controlled trial that assessed the impact of 2 different IP chemotherapy regimens on PFS among women with newly diagnosed advanced ovarian cancer (9). Patients were randomly assigned to 6 cycles of IV dose-dense paclitaxel $\left(80 \mathrm{mg} / \mathrm{m}^{2}\right)$ with IV carboplatin (IV carboplatin arm) versus IV dose-dense paclitaxel $\left(80 \mathrm{mg} / \mathrm{m}^{2}\right)$ with IP carboplatin (IP carboplatin arm) versus a modified GOG-172 regimen with a 3 -hour infusion of IV paclitaxel $\left(135 \mathrm{mg} / \mathrm{m}^{2}\right)$ on day 1, IP cisplatin $\left(75 \mathrm{mg} / \mathrm{m}^{2}\right)$ on day 2 , and then IP paclitaxel $\left(60 \mathrm{mg} / \mathrm{m}^{2}\right)$ on day 8 (IP cisplatin arm). All participants received additional therapy with bevacizumab $(15 \mathrm{mg} / \mathrm{kg})$ during cycles 2-6 and as maintenance through cycle 22 and beyond. Walker et al. reported no differences in outcome between the 3 groups, with a median PFS of 24.9 months in the IV carboplatin arm, 27.4 months in the IP carboplatin arm, and 26.2 months in the IP cisplatin arm. The authors suggested that the additional therapy with bevacizumab and the lower dose of cisplatin used for the study might explain the negative findings of the trial. However, the administration of IP cisplatin had already demonstrated higher toxicity than that of the standard IV carboplatin arm in their trial. A further increase in cisplatin dosage, as high as that of GOG-172, would be associated with even higher systemic side effects; therefore, the potential benefit of such a regimen remains very questionable. The results of GOG-252 have led to a change in clinical practice, and postoperative IP chemotherapy has been widely abandoned and is currently not considered a standard first-line therapy.

\section{HIPEC for ovarian cancer}

HIPEC differs markedly from IP chemotherapy in the postoperative setting, as it constitutes a single procedure in which heated chemotherapy $\left(42-43{ }^{\circ} \mathrm{C}\right)$ is administered into the peritoneal cavity at the end of cytoreductive surgery. During HIPEC, the chemotherapeutic agent is usually administered either via the "open coliseum technique" or "closed abdomen technique". Some retrospective data suggest that higher concentrations of the chemotherapeutic drug within the tissues and a more homogenous distribution within the abdominal cavity can be achieved with the open technique, while the closed technique appears to be associated with more stable IP temperature and increased drug penetration into the tissue $(10,11)$. However, there are no prospective, randomized trial data that have compared the effectiveness of the different techniques, and therefore, a clear recommendation to use one over the other cannot be given.

At Memorial Sloan Kettering Cancer Center (MSK), we use the "closed abdomen technique", as we feel it more consistently keeps the perfusion temperature over $42{ }^{\circ} \mathrm{C}$ and provides the greatest protection for surgeons and operating 
room personnel by minimizing their exposure to the chemotherapeutic drug. In addition, several experimental studies have suggested increased drug penetration into the tissue due to higher intra-abdominal pressure with the closed technique $(12,13)$. The potential shortcomings of the closed technique, such as the lack of direct control of the abdominal cavity or possible suboptimal heat and chemotherapy distribution, have not been of major concern at our institution.

\section{HIPEC for primary advanced ovarian cancer}

Most of the scientific evidence for HIPEC in primary advanced ovarian cancer is based on several retrospective, observational case-cohort studies that have reported mixed oncologic outcomes.

A systematic review published by Chiva et al. (14) in 2015 failed to show a survival benefit for patients treated with HIPEC at the time of primary debulking surgery. For the final analysis, 11 publications totaling 248 patients with advanced primary ovarian cancer were reviewed. The median OS and disease-free survival were 37.3 and 14.4 months, respectively, similar to outcomes reported in previously published studies that examined standard approaches without HIPEC. However, 2 recently conducted systematic reviews and meta-analyses reported improved outcomes after cytoreduction and HIPEC in patients with primary advanced ovarian cancer. In a metaanalysis by Huo et al. (15), 9 comparative studies and 28 studies examining HIPEC during cytoreductive surgery for primary and/or recurrent epithelial ovarian cancer were included. In primary epithelial ovarian cancer, cytoreductive surgery with HIPEC resulted in better oncologic outcomes compared with cytoreductive surgery alone, with an elevated hazard ratio (HR) of death for the cytoreductive surgeryalone cohort at 3 years (HR, 4.31; 95\% CI, 2.11-8.81) and 5 years (HR, 2.53; 95\% CI, 1.28-5.00). Similar findings were reported in a meta-analysis by Zhang et al. (16), with an OS benefit with the use of HIPEC in patients with primary epithelial ovarian cancer (HR, 0.59; 95\% CI, 0.46-0.72).

Aside from their retrospective design, these studies were limited by heterogenous patient populations and a large variety of chemotherapeutic regimens used, including single-agent platinum, non-platinum, and combination therapies. Even within a single study, variation in agents and doses were common, leading to difficulties in evaluating the clinical impact of the results. Therefore, survival outcomes in these studies should be interpreted with caution and require confirmation via prospective, randomized trials.

Prospective, randomized trials evaluating the use of HIPEC at the time of primary debulking surgery are still lacking. In a randomized, controlled trial from South Korea, Lim et al. (17) investigated the benefit of HIPEC at the time of primary or interval debulking surgery for patients with stage III-IV ovarian cancer. One-hundred eighty-four patients were randomized to HIPEC with IP cisplatin $\left(75 \mathrm{mg} / \mathrm{m}^{2}\right)$ for $90 \mathrm{~min}$ or no HIPEC (control arm). Randomization was performed intraoperatively based on residual tumor size $(<1 \mathrm{~cm})$. There were no mortalities, and postoperative morbidities were not statistically different between the two treatment arms, except for anemia $(67.4 \%$ vs. $50 \% ; \mathrm{P}=0.025)$ and creatinine elevation $(15.2 \%$ vs. $4.3 \% ; \mathrm{P}=0.026)$ in the HIPEC group. Survival analyses showed no superiority of the HIPEC arm, with 5 -year PFS rates of $20.9 \%$ and $16.0 \%(\mathrm{P}=0.569)$ and 5 -years $\mathrm{OS}$ rates of $51.0 \%$ and $49.4 \%(\mathrm{P}=0.574)$ in the HIPEC and control groups, respectively. However, subgroup analyses of oncologic outcomes for patients who underwent primary cytoreduction were not reported, and the final full manuscript has not been published yet. However, the study included patients who underwent both primary and interval debulking surgery, and the statistical power of the study was due to the relatively small sample size, probably potentially too small to detect a difference between the treatment groups. Several international, prospective phase 3 randomized, controlled trials have been planned or are already enrolling patients to determine whether there is a benefit with HIPEC in the primary debulking setting [NCT04280185, NCT03772028, NCT03373058].

In 2018, van Driel et al. published in the New England fournal of Medicine the findings of the first phase 3 randomized, controlled trial that evaluated the use of HIPEC with cisplatin at the time of interval debulking surgery for patients with stage III ovarian cancer who had undergone neoadjuvant IV chemotherapy (the OVHIPEC study) (18). In the study, 245 patients who had at least stable disease after 3 cycles of IV neoadjuvant paclitaxel $\left(170 \mathrm{mg} / \mathrm{m}^{2}\right)$ and carboplatin [area under the curve (AUC) $5-6 \mathrm{mg} / \mathrm{mL} / \mathrm{min}$ ] chemotherapy were randomly assigned to interval debulking surgery with or without HIPEC with IP cisplatin $\left(100 \mathrm{mg} / \mathrm{m}^{2}\right)$ perfusion for 90 minutes. Patients were randomized at the time of surgery following intraoperative assessment of resectability to no visible residual disease or minimal residual disease $(\leq 1 \mathrm{~cm}$ in largest diameter). Patients were given 3 more cycles of carboplatin 
and paclitaxel after surgey. Median PFS-the primary endpoint of the study - was 10.7 months for patients in the standard arm compared with 14.2 months for those in the HIPEC arm (HR, 0.66; 95\% CI, 0.50-0.87; P=0.003). At the time of analysis, $44 \%$ of patients were alive, and there was a significant median OS improvement for the HIPEC group (45.7 vs. 33.9 months; HR, 0.67; 95\% CI, 0.48-0.94; $\mathrm{P}=0.02)$. Grade 3-4 adverse events were similar between the two treatment arms ( $27 \%$ vs. $25 \%$; $\mathrm{P}=0.76)$. In patients who received HIPEC, postoperative chemotherapy was not delayed or compromised. Furthermore, healthrelated quality of life was similar between the two treatment arms. This was the first randomized trial in which the only intervention difference was the use of a single, 90-minute perfusion of cisplatin after surgery. The $100 \mathrm{mg} / \mathrm{m}^{2}$ dose of cisplatin was determined based on findings from phase 1/2 and retrospective studies. Standardized, protocol-specific surveillance, including computed tomography (CT) scans and CA-125 measurement, were used to assess the primary endpoint (PFS). Furthermore, the median PFS and OS in the control arm were similar to those of previous randomized clinical trials that included similar patients. Based on the trial data, a recent cost-effectiveness study showed that treatment with interval debulking surgery and HIPEC in patients with stage III ovarian cancer was associated with a substantial gain in quality-adjusted life-years. The incremental costeffectiveness ratio, expressed as euros per quality-adjusted life-year, was below the willingness-to-pay threshold in the Netherlands, indicating interval debulking surgery with HIPEC is cost effective for these patients. These results support the reimbursement of costs for patients treated with interval debulking surgery and HIPEC in countries with similar healthcare systems (19).

However, the study's results have been questioned by several authors due to an inappropriate initial sample size calculation, the potential bias by preoperative timing of randomization, the relatively small sample size of the study, the long recruitment period of over 9 years, and the higher toxicity rates for certain complications (i.e., infection, thromboembolism) in the HIPEC arm (20). Nevertheless, based on the positive results of the OVHIPEC trial, the National Comprehensive Cancer Network (NCCN) released updated guidelines in 2019 stating that HIPEC can be consider as a primary treatment option at the time of interval debulking surgery in patients with stage III disease treated with neoadjuvant chemotherapy.

At MSK, we recently implemented the use of cytoreductive surgery with HIPEC for patients undergoing interval debulking using the van Driel protocol (18). Of note, we do not administer HIPEC to patients with a creatinine level $>1.5 \mathrm{mg} / \mathrm{dL}(\mathrm{CrCl}<30 \mathrm{~mL} / \mathrm{min})$ and/ or insulin-dependent diabetes mellitus. Furthermore, we recommend special consideration for patients at higher risk for toxicity, such as patients with a preoperative creatinine level of $1.0-1.5 \mathrm{mg} / \mathrm{dL}(\mathrm{CrCl}<50 \mathrm{~mL} / \mathrm{min})$, non-insulin dependent diabetes mellitus, baseline neuropathy, or baseline hearing loss. In these patients, a dose reduction (cisplatin $80 \mathrm{mg} / \mathrm{m}^{2}$ ) or even avoidance of HIPEC should be considered. We administer preoperative IV palonosetron (250 mcg), aprepitant (130 mg), and dexamethasone (12 mg) to all patients undergoing HIPEC. The dose, duration, and time of administration of pre-medications and antiemetics may be modified as clinically indicated. Prior to HIPEC administration, sufficient fluid therapy (normal saline $500 \mathrm{~mL} / \mathrm{h}$ ) and a single IV bolus of mannitol should be given. Postoperatively, close follow-up, especially of urine output with avoidance of nephrotoxic medications, is necessary.

\section{HIPEC for recurrent ovarian cancer}

Similar to the upfront setting, the majority of data evaluating HIPEC in recurrent ovarian cancer with platinum-sensitive (21-23) or -resistant disease (24-26) has been based on retrospective studies.

Results from the first prospective phase 3 randomized, controlled trial of HIPEC in the treatment of recurrent ovarian cancer were published by Spiliotis et al. in 2015 (27). In the study, 120 patients with both platinum-sensitive and platinum-resistant disease were treated with conventional secondary cytoreductive surgery with or without HIPEC. IP cisplatin $\left(75 \mathrm{mg} / \mathrm{m}^{2}\right)$ and paclitaxel $\left(175 \mathrm{mg} / \mathrm{m}^{2}\right)$ were administered to patients with platinum-sensitive disease, and IP doxorubicin $\left(35 \mathrm{mg} / \mathrm{m}^{2}\right)$ and paclitaxel $\left(175 \mathrm{mg} / \mathrm{m}^{2}\right)$ or mitomycin $\left(15 \mathrm{mg} / \mathrm{m}^{2}\right)$ were administered to patients with platinum-resistant disease. Patients randomized to HIPEC demonstrated improved survival compared to patients in the standard group (27 vs. 13 months, respectively; $\mathrm{P}<0.006$ ), with the greatest benefit seen in patients with platinumresistant disease. Complete cytoreduction was associated with longer survival, and patients with a peritoneal cancer index score of $<15$ also appeared to have improved outcomes. However, the validity of this prospective study has been sharply criticized by several authors. Besides the drawbacks with the statistical analysis of the study, the main points of criticism were the potential bias induced by the 
preoperative randomization, the heterogeneity of patient cohorts and chemotherapeutic regimens used, and the missing specifications of postoperative chemotherapy and follow-up $(28,29)$.

Our group at MSK recently reported preliminary data from a phase 2 randomized, controlled trial evaluating secondary cytoreductive surgery with or without HIPEC in patients with recurrent, platinum-sensitive ovarian cancer (30). After secondary cytoreductive surgery to $\leq 0.5 \mathrm{~cm}$ visible residual disease, patients were intraoperatively randomized to carboplatin HIPEC $\left(800 \mathrm{mg} / \mathrm{m}^{2}\right.$ for 90 minutes) or no HIPEC. A total of 98 patients were included, of whom 49 were randomized to the HIPEC group. There were no statistically significant differences in median PFS (15.4 vs. 12.3 months, $\mathrm{P}=0.17)$ or median OS (69.2 vs. 53.1 months, $\mathrm{P}=0.32$ ) between the two study arms. Of note, there were no perioperative mortalities, and no increased perioperative morbidity or toxicity was seen with the use of HIPEC. Based on this trial, we concluded that secondary cytoreductive surgery with carboplatin HIPEC followed by 5 cycles of platinum-based chemotherapy is not superior to secondary cytoreductive surgery without HIPEC followed by 6 cycles of platinum-based chemotherapy. Therefore, HIPEC after secondary cytoreductive surgery should only be offered to patients in the setting of a prospective clinical trial.

\section{Conclusion}

Cytoreductive surgery with HIPEC is a safe intervention in patients with ovarian cancer and should be offered as standard of care to patients with International Federation of Gynecology and Obstetrics (FIGO) stage III disease undergoing interval debulking surgery. Outside the clinical trial setting, cisplatin should be used at a dose of $100 \mathrm{mg} / \mathrm{m}^{2}$. Due to potential side effects and morbidity, this treatment should be offered only to women who do not have major comorbidities, especially pre-existing renal disease or impairment. In all other settings, HIPEC is still considered experimental. Postoperative IP chemotherapy in ovarian cancer remains controversial, particularly after the findings of GOG-252, and should be offered to patients only in the setting of clinical trials.

\section{Acknowledgments}

Funding: Drs. Zivanovic and Chi are supported in part through the NIH/NCI Cancer Center Support Grant P30

\section{CA008748.}

\section{Footnote}

Provenance and Peer Review: This article was commissioned by the Guest Editors (Paul H. Sugarbaker and Kurt Van der Speeten) for the focused issue"Intraperitoneal Chemotherapy for Peritoneal Metastases: HIPEC, EPIC, NIPEC, PIPAC and More" published in Fournal of Gastrointestinal Oncology. This article has undergone external peer review.

Reporting Checklist: The authors have completed the Narrative Review Checklist. Available at http://dx.doi. org/10.21037/jgo-20-274

Conflicts of Interest: All authors have completed the ICMJE uniform disclosure form (available at http://dx.doi. org/10.21037/jgo-20-274). The focused issue was sponsored by the Peritoneal Surface Oncology Group International (PSOGI). Outside the submitted work, Dr. Chi reports personal fees from Bovie Medical Co., Verthermia Inc. (now Apyx Medical Corp.), C Surgeries, and Biom 'Up, as well as recent stock ownership in Intuitive Surgical, Inc. and TransEnterix, Inc. (sold 12/18). The authors have no other conflicts of interest to declare.

Ethical Statement: The authors are accountable for all aspects of the work in ensuring that questions related to the accuracy or integrity of any part of the work are appropriately investigated and resolved.

Open Access Statement: This is an Open Access article distributed in accordance with the Creative Commons Attribution-NonCommercial-NoDerivs 4.0 International License (CC BY-NC-ND 4.0), which permits the noncommercial replication and distribution of the article with the strict proviso that no changes or edits are made and the original work is properly cited (including links to both the formal publication through the relevant DOI and the license). See: https://creativecommons.org/licenses/by-nc-nd/4.0/.

\section{References}

1. Ferlay J, Soerjomataram I, Ervik M, et al. GLOBOCAN 2012 v1. 0, Cancer Incidence and Mortality Worldwide: IARC CancerBase No. 11 [Internet]. [cited 2015 July 30].

2. Torre LA, Islami F, Siegel RL, et al. Global Cancer 
in Women: Burden and Trends. Cancer Epidemiol Biomarkers Prev 2017;26:444-57.

3. Buys SS, Partridge E, Black A, et al. Effect of Screening on Ovarian Cancer Mortality: The Prostate, Lung, Colorectal and Ovarian (PLCO) Cancer Screening Randomized Controlled Trial. JAMA 2011;305:2295-303.

4. Stordal B, Davey MJIl. Understanding cisplatin resistance using cellular models. IUBMB Life 2007;59:696-9.

5. Alberts DS, Liu P, Hannigan EV, et al. Intraperitoneal cisplatin plus intravenous cyclophosphamide versus intravenous cisplatin plus intravenous cyclophosphamide for stage III ovarian cancer. N Engl J Med 1996;335:1950-5.

6. Armstrong DK, Bundy B, Wenzel L, et al. Intraperitoneal cisplatin and paclitaxel in ovarian cancer. New Engl J Med 2006;354:34-43.

7. Markman M, Bundy BN, Alberts DS, et al. Phase III trial of standard-dose intravenous cisplatin plus paclitaxel versus moderately high-dose carboplatin followed by intravenous paclitaxel and intraperitoneal cisplatin in small-volume stage III ovarian carcinoma: an intergroup study of the Gynecologic Oncology Group, Southwestern Oncology Group, and Eastern Cooperative Oncology Group. J Clin Oncol 2001;19:1001-7.

8. Suidan RS, Zhou Q, Iasonos A, et al. Prognostic significance of the number of postoperative intraperitoneal chemotherapy cycles for patients with advanced epithelial ovarian cancer. Int J Gynecol Cancer 2015;25:599-606.

9. Walker JL, Brady MF, Wenzel L, et al. Randomized Trial of Intravenous Versus Intraperitoneal Chemotherapy Plus Bevacizumab in Advanced Ovarian Carcinoma: An NRG Oncology/Gynecologic Oncology Group Study. J Clin Oncol 2019;37:1380-90.

10. Elias D, Antoun S, Raynard B, et al. Treatment of peritoneal carcinomatosis using complete excision and intraperitoneal chemohyperthermia. A phase I-II study defining the best technical procedures. Chirurgie 1999;124:380-9.

11. Ortega-Deballon P, Facy O, Jambet S, et al. Which method to deliver hyperthermic intraperitoneal chemotherapy with oxaliplatin? An experimental comparison of open and closed techniques. Ann Surg Oncol 2010;17:1957-63.

12. Esquis P, Consolo D, Magnin G, et al. High intraabdominal pressure enhances the penetration and antitumor effect of intraperitoneal cisplatin on experimental peritoneal carcinomatosis. Ann Surg 2006;244:106.

13. Ferron G, Gesson-Paute A, Classe JM, et al. Feasibility of laparoscopic peritonectomy followed by intra-peritoneal chemohyperthermia: an experimental study. Gynecol Oncol 2005;99:358-61.

14. Chiva LM, Gonzalez-Martin A. A critical appraisal of hyperthermic intraperitoneal chemotherapy (HIPEC) in the treatment of advanced and recurrent ovarian cancer. Gynecol Oncol 2015;136:130-5.

15. Huo YR, Richards A, Liauw W, Morris DL. Hyperthermic intraperitoneal chemotherapy (HIPEC) and cytoreductive surgery (CRS) in ovarian cancer: A systematic review and meta-analysis. Eur J Surg Oncol 2015;41:1578-89.

16. Zhang G, Zhu Y, Liu C, et al. The Prognosis Impact of Hyperthermic Intraperitoneal Chemotherapy (HIPEC) Plus Cytoreductive Surgery (CRS) in Advanced Ovarian Cancer: The Meta-Analysis. J Ovarian Res 2019;12:33.

17. Lim MC, Chang SJ, Yoo HJ, et al. Randomized trial of hyperthermic intraperitoneal chemotherapy (HIPEC) in women with primary advanced peritoneal, ovarian, and tubal cancer. J Clin Oncol 2017;35:5520.

18. van Driel WJ, Koole SN, Sikorska K, et al. Hyperthermic Intraperitoneal Chemotherapy in Ovarian Cancer. N Engl J Med 2018;378:230-40.

19. Koole SN, van Lieshout, van Driel WJ, et al. Cost Effectiveness of Interval Cytoreductive Surgery With Hyperthermic Intraperitoneal Chemotherapy in Stage III Ovarian Cancer on the Basis of a Randomized Phase III Trial. J Clin Oncol 2019;37:2041-50.

20. Vergote I, Harter P, Chiva L. Hyperthermic intraperitoneal chemotherapy does not improve survival in advanced ovarian cancer. Cancer 2019;125:4594-7.

21. Deraco M, Virzi S, Iusco DR, et al. Secondary cytoreductive surgery and hyperthermic intraperitoneal chemotherapy for recurrent epithelial ovarian cancer: a multi-institutional study. BJOG 2012;119:800-9.

22. Bakrin N, Bereder JM, Decullier E, et al. Peritoneal carcinomatosis treated with cytoreductive surgery and Hyperthermic Intraperitoneal Chemotherapy (HIPEC) for advanced ovarian carcinoma: a French multicentre retrospective cohort study of 566 patients. Eur J Surg Oncol 2013;39(:1435-43.

23. Classe JM, Glehen O, Decullier E, et al. Cytoreductive Surgery and Hyperthermic Intraperitoneal Chemotherapy for First Relapse of Ovarian Cancer. Anticancer Res 2015;35:4997-5005.

24. Fagotti A, Costantini B, Petrillo M, et al. Cytoreductive surgery plus HIPEC in platinum-sensitive recurrent ovarian cancer patients: a case-control study on survival in patients with two year follow-up. Gynecol Oncol 
2012;127:502-5.

25. Cascales-Campos PA, Gil J, Gil E, et al. Treatment of microscopic disease with hyperthermic intraoperative intraperitoneal chemotherapy after complete cytoreduction improves disease-free survival in patients with stage IIIC/ IV ovarian cancer. Ann Surg Oncol 2014;21:2383-9.

26. Munoz-Casares FC, Medina-Fernandez FJ, ArjonaSanchez A, et al. Peritonectomy procedures and HIPEC in the treatment of peritoneal carcinomatosis from ovarian cancer: Long-term outcomes and perspectives from a high-volume center. Eur J Surg Oncol 2016;42:224-33.

27. Spiliotis J, Halkia E, Lianos E, et al. Cytoreductive surgery and HIPEC in recurrent epithelial ovarian cancer: a prospective randomized phase III study. Ann Surg Oncol 2015;22:1570-5.

28. Harter P, Reuss A, Sehouli J, et al. Brief Report About the

Cite this article as: Boerner T, Zivanovic O, Chi DS. Narrative review of cytoreductive surgery and intraperitoneal chemotherapy for peritoneal metastases in ovarian cancer. J Gastrointest Oncol 2021;12(Suppl 1):S137-S143. doi: 10.21037/ jgo-20-274
Role of Hyperthermic Intraperitoneal Chemotherapy in a Prospective Randomized Phase 3 Study in Recurrent Ovarian Cancer From Spiliotis et al. Int J Gynecol Cancer 2017;27:246-7.

29. Harter P, du Bois A, Mahner S, et al. Statement of the AGO Kommission Ovar, AGO Study Group, NOGGO, AGO Austria and AGO Switzerland Regarding the Use of Hyperthermic Intraperitoneal Chemotherapy (HIPEC) in Ovarian Cancer. Geburtshilfe Frauenheilkd 2016;76:147-9.

30. Zivanovic O, Chi D, Zhou Q, et al. A randomized phase II trial of secondary cytoreductive surgery (SCS) +/carboplatin hyperthermic intraperitoneal chemotherapy (HIPEC) in patients (pts) with recurrent platinum-sensitive ovarian cancer (EOC). J Clin Oncol 2020;38:6016. 